\title{
A Churn-Resistant Strategy for a Highly Reliable P2P System
}

\author{
Giscard Wepiwé ${ }^{1}$ and Sahin Albayrak ${ }^{2}$ \\ 1 Technische Universität Berlin, DAI-Labor \\ http://www.dai-labor.de \\ 2 Faculty of Electrical Engineering and Computer Science, \\ Franklinstr. 28/29, D-10587 Berlin, Germany \\ \{giscard.wepiwe, sahin.albayrak\}@dai-labor.de
}

\begin{abstract}
This paper proposes a churn-resistant strategy designed on top of a highly reliable P2P overlay network [1, with degre $112 \Delta+2$, where $\Delta$ is the degree of a De Bruijn digraph [2]. We show that when each node in the network periodically retransmits only one KEEPALIVE message to one of its neighbors in the network, any node's failure can be detected within an optimal timeout. As a major contribution, we demonstrate that even in failure situations the lookup of any available resource is achieved with the lowest possible maintenance overhead $O(1)$ along the shortest path of length $D_{C M R}=\log _{\Delta}(N(\Delta-1)+\Delta)-1$ with $N$ being the maximal number of nodes in the network.
\end{abstract}

\section{Introduction}

The proliferation of Internet-scale services and the advent of peer-to-peer (P2P) applications for data sharing have brought about considerable attention to the resource distribution and lookup problem in dynamic distributed computer environments such as $\mathrm{P} 2 \mathrm{P}$ systems. An important characteristic of $\mathrm{P} 2 \mathrm{P}$ systems is the dynamic nature of their nodes, which are in a continuous process of "join and leave". The result is a topology changing network where resource management issue is a highly challenging one.

Studies on dynamic graph theory [3] have shown that low-degree networks suffer from network disconnection when even only one node is deleted. On the other hand, networks of large degrees are indeed robust but susceptible to collapse as some nodes collectively fail. Moreover, it is a fact that fast detection of failures enables rapid recovery and contributes to keep the network in a stable state. This means that the frequent retransmission of periodic information to the neighborhood can significantly reduce the failure detection time. However, frequent retransmission of presence information (also called KEEPALIVE message in this paper) at high rate to neighbors would increase control overhead

${ }^{1}$ The degree of a vertex $\Delta(x)$ is the number of vertices adjacent to $x$. The degree of a graph $\mathrm{G}$ is $\Delta_{G}=\max \{\Delta(x), x \in V\}$. With $\mathrm{G}$ defined as $G=(V, E)$, where $\mathrm{V}$ is the set of vertex and $\mathrm{E}$ is the set of Edge of $\mathrm{G}$. 
in the network. Although several P2P proposals for churn management have been proposed recently to deal with these issues [4, 5, 6, 7, 8, many fundamental questions remain unanswered. Some of these are:

- How to conceive network maintenance strategies such that the deletion of a node or a subset of nodes does not cause the network to become disconnected or to collapse.

- How to choose the timeout for failure detection such that (i) the susceptibility to use the alternative routing solution is kept low and (ii) the control overhead and the message lost are minimized.

In what follows, we propose a churn-resistant strategy which responds to these open questions. The strategy is to be deployed on top of a highly reliable P2P network overlay, which is presented briefly in the Section 2. Interested readers may refer to [1] for more details.

\section{Overview of the Concentric Multi-ring Overlay (CMR)}

The churn-resistant strategy presented in this paper is designed to detect and recover failure in a $\mathrm{P} 2 \mathrm{P}$ network based on the $\mathrm{CMR}$ overlay. For convenience, we give an overview of the CMR network model in this section.

\subsection{System Model}

A CMR-based network is organized as a concentric multi-ring representation, where each of the $N$ nodes $-N$ as the total number of nodes in the system - are interconnected with $2 \Delta+2$ neighbors as shown in Figure 1. The CMR representation consists of several concentric rings, numbered with ring identifier (RID) from 1 for the innermost ring to $D_{\max }$ for the outermost ring. Nodes on a ring with identifier $D$ are interconnected so to form a De Bruijn graph 2] with diameter $2 D$ and degree $\Delta$. Each node in the network is assigned an identifier (ID) and each ring consists of $\Delta^{D}$ nodes. The node's ID is a $\Delta$-base number with $D$ digits; e.g the node $x=213121$ is a 4-base integer located on the ring with identifier 6 .

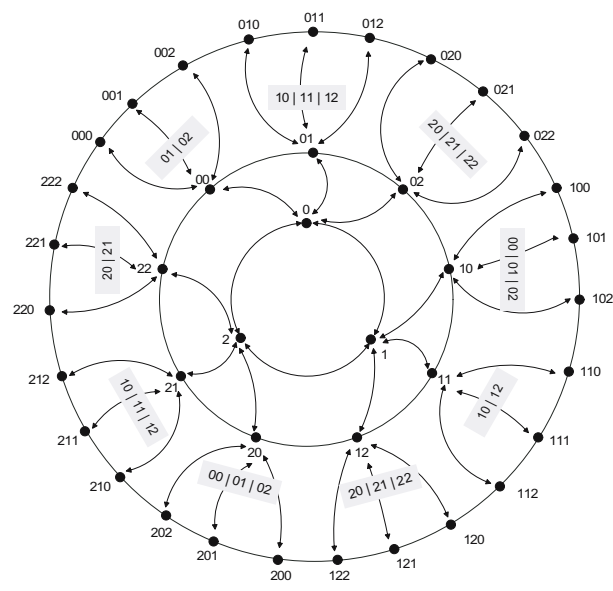

Fig. 1. The Logical Representation of Nodes on the Concentric Multi-ring Overlay

According to the dynamic behavior of the P2P systems, the network can grow as necessary. Nodes always join

${ }^{2}$ Diameter of a graph is defined as the minimum distance between the two most distant nodes in the network. 
the overlay network on its outermost ring. The ring's construction always begins with the node $x_{\{\text {init,D }}$ having the lowest identifier on the ring; where $x_{\{\text {init }, D\}}=x_{D-1} x_{D-2} \ldots x_{1} x_{0}$, with $x_{i}=0$ and $i \in\{0,1, \ldots, D-1\}$. The total number of nodes in the network is at most $N_{\max }=\frac{\Delta^{D+1}-\Delta}{\Delta-1}$ with $\Delta>1$.

In this work, we choose to interconnect nodes on the same ring after the De Bruijn principles owing to two reasons: (i) nodes in a De Bruijn network require no more than $\Delta$ neighbors to ensure routing in the shortest path; (ii) the routing distance between any two peers in a De Bruijn network is not longer than $\log _{\Delta} N$ which is closer to the Moore bound 9 3 than many other solutions based on Distributed Hash Table like Chord $(\log N)$ for example. However, when nodes join and leave continually in the system, there is a need to design management strategy in order to resist to churn and continue to route along the shortest path. The questions on how a failure is detected and how it is recovered, are detailed later on in Section 3.

\subsection{Routing Scheme}

Routing in our CMR network is an extension of the De Bruijn routing, where each node can forward a message toward its neighbor on the way to the destination. Each node $x$ in a CMR ring with identifier $D$ has at most $2 \Delta+2$ neighbors, where messages can be routed depending on the destination ID; these are (i) the $\Delta$ nodes $y=x_{D-1} x_{D-2} \ldots x_{1} \beta$ on the same ring; (ii) the $\Delta$ nodes $y=$ $x_{D-1} x_{D-2} \ldots x_{1} x_{0} \beta$ on the next outer ring; (iii) the node $y=x_{D-1} x_{D-2} \ldots x_{1}$ on the next inner ring; and (iv) the head node $y=0 x_{D-1} x_{D-2} \ldots x_{1}$ or $y=$ $0 x_{D-1} x_{D-2} \ldots x_{2}$ if $x$ is the ring contact node, with $0 \leq \beta \leq \Delta-1$.

Each node $x$ in the network maintains at most 2 tables:

- A routing table with the List_1 of pairs (NodeID, IP Address) for maintaining information about the De Bruijn neighbors and the List_2 of pairs (NodeID, IP Address) for maintaining information about the outer ring, inner ring neighbors and the head node.

- A resource location table with the list of pairs (GUIDE, Provider's IP Address). Each resource is represented in the network with a Global Unique IDEntifier (GUIDE) which is a $\Delta$-base integer.

To route between any two peers $x$ and $y$ in the network, a next-hop is chosen on-demand at each step on the way to the destination. Results in [1] show that routing between any two nodes in the network can be achieved within at most $D_{C M R}+1=\log _{\Delta}(N(\Delta-1)+\Delta)$ overlay hops.

\subsection{Resource Distribution Scheme}

The resource distribution scheme specifies how resources are distributed among the peers in the network. Each node $x$ on ring with ID $D_{x}$ can place information

\footnotetext{
${ }^{3} \log _{\Delta}(N(\Delta-2)+2)-\log _{\Delta-1} \Delta$ with $\Delta>2$.
} 
about its resource $R$ of length $L$ at any node $y=R \div \Delta^{L-D_{x}}$ on the same logical ring with ID $D_{x}$ and at any node $y=R \div \Delta^{L-D_{x}+1}$ on the next inner ring 5 with identifier $D_{x}-1$. As the network grows, the resource placement is extended to all nodes $y=R \div \Delta^{L-D_{x}-i}$, with $i \in\left\{1,2, \ldots, D_{\max }-D_{x}\right\}$ on the outer rings. In this paper, node $y$ indicates the ambassador of $x$ for the resource $R$ in the network.

Lemma 1. For any resource $R$, each node in the network has at least 1 and at most $D_{\max }$ ambassadors in the network. Where $N \geq \Delta$ and $D_{\max }$ being the total number of rings forming the network.

Proof. $R \div \Delta^{L-D_{x}}$ is the ID of exactly one node on the same ring as $x$, when $N \geq \Delta$. As the network grows, the outer rings are constructed and there is at most one more node with ID equal to $R \div \Delta^{L-D_{x}-i}$ when $0<i<D_{\max }-D_{x}$. Thus, in a network with a total of $D_{\max }$ rings, each node $x$ has an ambassador for its resource $R$ on all the $D_{\max }-D_{x}+1$ rings with ring identifier $A$, so that $A \geq D_{x}-1$.

Hence, each node $x$ has at most $D_{\max }$ ambassadors in the network.

\subsection{Resource Lookup Scheme}

As resources are always distributed from the inner to the outer ring, a node $z$ on ring $D_{z}$ looking for a resource $R$ of length $L=|R|$ provided by $x$ sends a resource lookup request to the node $\bar{x}=R \div \Delta^{L-D_{z}}$. With a high probability, node $\bar{x}$ knows $x$, a provider of $R$. The node $\bar{x}$ and the node on the way to $x$ forwards the message to their outer ring until a possible node $y$ is reached that knows the provider of the resource $R$ or until the provider itself is reached.

Theorem 1. Resource lookup in the network can find an ambassador of the resource provider in at most $D_{C M R}$ hops.

Proof. If a resource $R$ exists in the network, its furthest provider can be located after at most $D_{C M R}+1$ forwarding hops as stipulated in Section 2.2. If a provider is located on the outermost ring with identifier $D_{C M R}$, then according to Lemma 1 there is a node $y$ on the ring with identifier $D_{C M R}-1$ which is ambassador for $R$. To route from any ring in the network to the ring with identifier $D_{C M R}-1$, one needs at most $D_{C M R}$ hops. In conclusion, a node $y=R \div \Delta^{L-D_{C M R}+1}$ is reached after at most $D_{C M R}$ hops, which has a location table entry with the resource $R$.

\section{Network Maintenance}

A CMR-based network is in a consistent state if and only if all the inner rings are fully constructed and each node on the outermost ring knows its head node.

\footnotetext{
${ }^{4}$ It is assumed that $L>D$.

${ }^{5} \div$ is used for the division of two $\Delta$-base integers.
} 
The head node of a node $x$ is the node to which $x$ regularly sends KEEPALIVE message. In this manner, the nodes in the network must be able to detect node's failure in their neighborhood in order to start recovery procedure and bring back the network to a consistent state.

\subsection{Node Arrival}

To join the network, a node must know the IP address of at least one node already in the network - the so-called "network contact node". In the following description, we assume that a node $x$ willing to join the network is aware of at most one network contact node. In order to join however, the node $x$ should: (i) determine the identifier of the outermost ring and (ii) get a node's ID from the network.

Determine the actual outermost ring: A joining node $x$ sends a "join request" message to a network contact node, asking for the attribution of an ID on the actual outermost ring. The "join request" message is routed to the node $x_{\{\text {init,D\} }}$ on the outermost ring, which ensures that an ID is assigned to $x$. If the outermost ring is full, then a new outer ring is created and $x$ becomes the node $x_{\{\text {init, } D+1\}}$ on that ring, else the newcomer is attributed an identifier in the middle of the next larger space on the outermost ring. The operation of determining the actual outermost ring may involve at most $D$ nodes to forward the "join request" message.

Determine the ID of a node joining the network: To better illustrate the node joining procedure, let's have a look of the abstract ring representation in form of a tree in Figure 2 .

To ensure an even distribution of keys at all times in the system, the outermost ring's contact node has $\Delta-1$ branches with well-defined branch size. The branch size

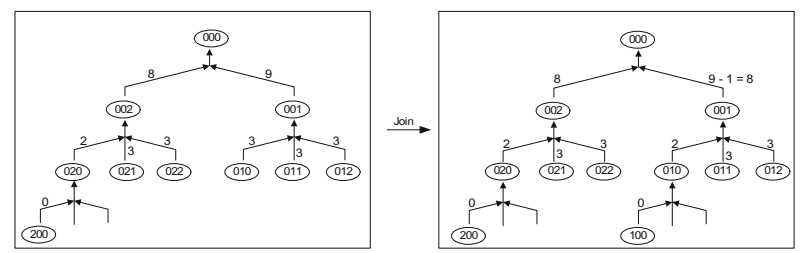

Fig. 2. Tree representation of the ring with $R I D=3$

decreases as a new node is inserted on the ring. The original size of a branch is computed using the formula branchsize $=\frac{\Delta^{D-\text { index }}-1}{\Delta-1}$ with index being the position of the first occurrence of a non-zero digit from the left of the node's ID; for example the original size of the 3 branches of the node 020 is 1 . After each "join request" message, the outermost ring's contact node forwards the message to the branch that has the highest branch size and decreases the related branch size by one. A new ring is created, if all the branches of the ring contact node have a size being equal to zero. The head node is the node who sent the "join invite" message to the newcomer node. Hence, after joining the system the new node notifies its presence to its neighbors, distributes its resources, and 
collects notification messages received from others nodes to construct its tables. After this, the node is connected and can request and exchange resources from respectively with the rest of the network.

Each node joining the network generates one message to determine the ID of outermost ring, one resource location message to distribute each of its resources, and at most $\Delta+1$ messages to update its routing table. This makes a total number of $\Delta+3$ messages involving at most $D$ nodes for forwarding.

\subsection{Failure Detection and Recovery}

In order to keep the network in a consistent state, the failures of nodes due to e.g. sudden or intentional departures should be detected, and their effect on message routing and resource lookup operations in the network should be minimized.

Network Failure Detection: In order to still aware of the presence of their neighbors, any node $x$ on the logical overlay network informs after a time period $T$ its head node denoted as $x^{\text {head }}$ about its presence in the network. The choice of $T$ is conditioned by the dynamic pattern of the network. In this work, we consider two cases of node departure in the network. When a node $x$ voluntary leaves the network, it informs a successor about its intention to leave. However, when a node has been deleted from the network by a malicious actor for example, its head neighbor must automatically detect its absence after a given timeout. That is, when the head neighbor of a node $x$ does not receive any message from $x$ after a timeout period Tout $=f(T)$, then $x$ has probably crashed. The study presented in 4 demonstrates that the choice of the timeout value Tout is a significant factor for the network reliability and hence for the lookup latency under churn. For our network simulation, we choose the style of timeout calculation as in mature TCP works such as [10] for message retransmission during network congestion. Hence, we choose

$$
\text { Tout }=T+\frac{A V G}{2}+4 \times V A R,
$$

where $A V G$ is the observed average round-trip time and $V A R$ is the observed mean variance of that time; $T$ is derived from experimental network measurement study of a KaZAa network [11. That is, after each period Tout, if the head neighbor does not receive any message from $x$, then the node is assumed as probably failed. We say probably, because it may happen that $x$ is still available in the network but the link between $x$ and $x^{\text {head }}$ is congested. In this work, we assume that the absence of a KEEPALIVE message between two nodes $x$ and $x^{\text {head }}$ is a sign that $x$ has failed.

The Network Recovery Procedure: Once a node $x^{\text {head }}$ has detected the failure of a node $x$, two operations are processed. First, node $x^{\text {head }}$ sends a "route obsolete message" to all the nodes, who know $x$ as a forwarding hop. On receipt of a "route obsolete message", nodes update their routing table. Secondly, node $x^{\text {head }}$ chooses a successor for the node $x$. Therefore, it sends a "choose 
successor" message to the outermost ring's contact node, which forwards it to the smallest leaf on the tree representation of the ring. The smallest leaf leaves the outermost ring and replaces the failed node on the inner ring. Once on the new ring with a new ID, the node subsequently informs its ongoing De Bruijn neighbors on the same ring. It also informs its inner ring's neighbor and its $\Delta$ neighbors on the next outer ring about its new IP address. This stabilization process enables the network to converge very fast and generates only $2 \Delta+2$ messages.

Further, the new node $x$ chooses one ambassador for each of its resource.

\subsection{Concurrent JOIN and LEAVE}

We also study the complexity of concurrent join and leave events in the network.

The concurrent JOIN protocol is similar to the isolated JOIN protocol with a difference on the join duration delay. That is, when $k$ nodes want to join the network simultaneously, their requests are processed subsequently and the join request can be delayed.

The concurrent LEAVE protocol is a challenging issue which basically relies on the isolated LEAVE protocol. After a malicious attack, several nodes on different rings could detect failure of their neighbor. In any case, the failure detection is done after a time $t$, with $0 \leq t \leq$ Tout. The recovery from a concurrent LEAVE event is a successive processing of the isolated network recovery procedure.

Additionally, if a request reaches a deficient node before the recovery from all failures is complete, the adaptive routing scheme is applied as described in Subsection 3.4 .

\subsection{Static Resilience Scheme}

The routing between any two nodes $x$ and $y$ in the network is a multi-hop forwarding operation as shown in Section 2.2. However, the routing operation presented there assumes that the network is in a consistent state and will consequently fail to route packets to its destination when a neighbor $z$ along the path from $x$ to $y$ suddenly fails. To address this issue, we propose a static resilience scheme which enables routing around the failure region.

When the computed next-hop node $z$ is not available in the table, the message is forwarded to the alternative node $\bar{x}$ which is chosen as follows:

- If the message target $y$ is a node on an outer ring and node $z$ is on the same ring than $x$, then $\bar{x}$ is the neighbor of $x$ on the next outer ring such that $\bar{x}$ is the node closest to the node $\operatorname{prefix}\left(D_{\bar{x}}, y\right)$ in the ID-space 6 .

- Else $\bar{x}$ is the head node

Theorem 2. Any resource request in the network is forwarded along the shortest path to its destination, even at high rate of node failure.

$\overline{{ }^{6} \operatorname{prefix}(i, y)}$ returns the first $i$ letters to the left of the word $y$. 
Proof. The static resilience scheme in this subsection ensures that a packet can be routed around a failure region. Here, we choose the most optimal next-hop for packet forwarding so that the routing operation as specified in Section 2.2 can still be applied. Additionally, we've shown that routing in the network is always achieved along the shortest path in the consistent network state. Thus, we can conclude that any request in the network is always forwarded along the shortest path to its destination even in failure situations.

\section{Performance Evaluation}

In this section, we evaluate the CMR protocol by means of a network emulation. The performance evaluation is given for high churn rate situation with little or no congestion. Our findings are based on nearly real network conditions as studied in [11. We emulate a network of about 1300 nodes running on 7 SUN Fire V210 machines with 2x1,0GHz UltraSPARC III 64 Bits with 2GB RAM; all the machines are connected with each other over a Gigabit Ethernet. We simulate node joining and leaving the network, resource distribution and resource request. During the simulation, one node is inserted in the network each two seconds. After the first five nodes have been initialized in the network, we start the churn situation at the rate of one failure or one leave after each five joins. While the network is growing, each node sends one KEEPALIVE message to its head node at a rate of one per 10 seconds and requests one arbitrary selected resource every 15 seconds.

In the sequel, we discuss some measurements results.

Lookup Path Length: At high rates of churn at least 99.5 percent of the 194109 requested resources are found by the requesters. Figure [3(a) shows that each successful lookup is achieved within $D_{C M R}=\log _{\Delta}(N(\Delta-1)+\Delta)-1$ hops and compares the average path length of CMR against Chord [5]; this result reveals that lookup in CMR is achieved within a lower path length than Chord at high rate of churn.

Load Balancing: We show in Figure 3(b) that even when many nodes come and go continually the available resources are fairly distributed between nodes in the network. The Figure 3(b) shows that the average distribution of resources per node is almost constant independently of the network size.

Routing Table Size: Figure 4(a) shows the evolution of the mean routing table size per node in a network. The routing table size is limited to maximum $2 \Delta+2$.

Failure Detection Delay and Recovery Delay: For our emulation, we set $T=10$ seconds for sending regular KEEPALIVE message. The graph of Figure 4(b) shows that the failure detection time mainly depends on $T$. The graph of Figure 4 (b) also presents the failure recovery delay, which is the time spent from the failure detection time until the recovery operation is complete. We can see that the failure recovery time is very short, depending on whether the failed node and the successor are on the same emulation machine or not. 


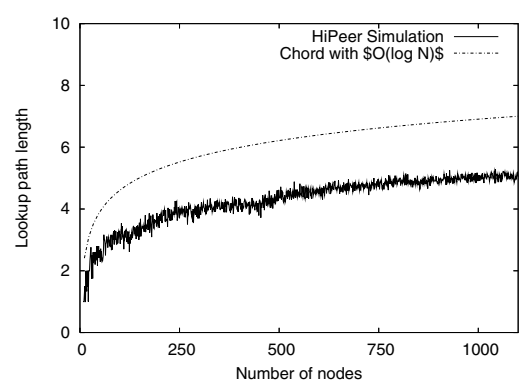

(a)

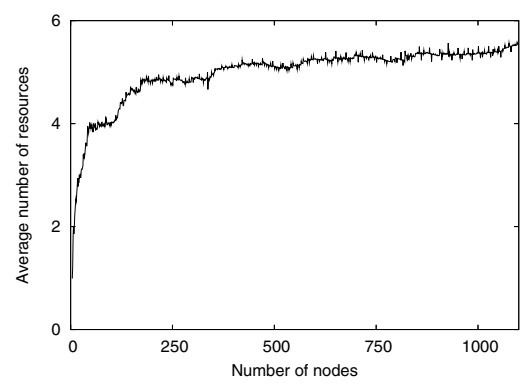

(b)

Fig. 3. (a) The average lookup path length of CMR with degree $\Delta=3$ compared to Chord. (b) The average number of resources stored per node in a failure-prone growing network.

\section{Related Work}

A plethora of different overlay networks with various interesting technical properties have been developed over the last five years 5 , 8. The protocols not only aim at defining scalable overlay structure for fast lookup of resources in P2P network environments but also address the failure resilience issue. In order to support resilience, they are mostly based on two principal classes of failure detection algorithms: (i) the reactive approach, where the nodes only send KEEPALIVE messages in data packets and (ii) the proactive approach, where the nodes periodically send KEEPALIVE messages to their neighbors. The reactive approach is generally used in the literature as an optimization of the active approach. Thus, many proposals for $\mathrm{P} 2 \mathrm{P}$ network resilience techniques such as [4, 6] rely on KEEPALIVE techniques to detect failure of peers in the network. The study of failure detection algorithms in [7] shows that the delay during failure detection is an important performance factor for resilience in $\mathrm{P} 2 \mathrm{P}$ systems; thus, it is

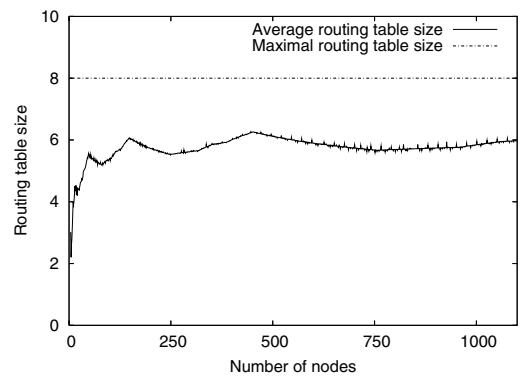

(a)

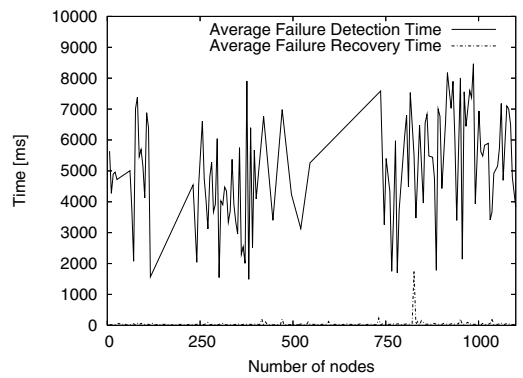

(b)

Fig. 4. (a)- The average failure detection and recovery time in a highly rate of network "join and leave". (b)- The average number of routing table entries per node as a function of the network size. 
worth to minimize the detection time of a node's failure. Moreover, in order to minimize the delay until the detection of a failure in the network further, some timeout calculation techniques are presented in [4].

\section{Conclusion}

We have presented a churn-resistant algorithm designed on top of a highly reliable concentric multi-ring overlay for management of large-scale highly dynamic P2P systems. We have proposed a network maintenance strategy where each node periodically retransmits only one control message to remain connected. A major contribution of the strategy is its ability to enable network consistency and resource lookup in at most $D_{C M R}=O\left(\log _{\Delta}(N(\Delta-1)+\Delta)-1\right.$ overlay hops even at high rate of churn with a lookup success percentage of about 99,5 .

\section{References}

1. Giscard Wepiwé and Plamen L. Simeonov. A concentric multi-ring overlay for highly reliable p2p systems. In Network Computing and Applications (NCA05), Cambridge, MA, USA, July 2005. IEEE Computer Society. To Appear.

2. N.G. de Bruijn. A combinatorial problem. In Nederl. Akad. Wetensh. Proc. 49, pages 768-764, 1946.

3. Ding-Zhu Du and D. Frank Hsu. Combinatorial Network Theory, volume 1 of 1948-III Series, chapter 3: De Bruijn Digraphs, Kautz Digraphs, and Their Generalizations, pages 65-105. Kluwer Academic Publishers. Printed in the Netherlands, 1996.

4. Sean Rhea, Dennis Geels, Timothy Roscoe, and John Kubiatowicz. Handling churn in a DHT. In Proceedings of the 2004 USENIX Annual Technical Conference (USENIX '04), Boston, Massachusetts, June 2004.

5. Ion Stoica, Robert Morris, David Karger, M. Frans Kaashoek, and Hari Balakrishnan. Chord: A scalable peer-to-peer lookup service for internet applications. SIGCOMM Comput. Commun. Rev., 31(4):149-160, 2001.

6. Simon S. Lam and Huaiyu Liu. Failure recovery for structured p2p networks: protocol design and performance evaluation. SIGMETRICS Perform. Eval. Rev., 32(1):199-210, 2004.

7. Shelley Zhuang, Dennis Geels, Ion Stoica, and Randy Katz. On failure detection algorithms in overlay networks. In INFOCOM'05, 2005. to appear.

8. Ben Y. Zhao, Ling Huang, Sean C. Rhea, Jeremy Stribling, Anthony D Joseph, and John D. Kubiatowicz. Tapestry: A global-scale overlay for rapid service deployment. IEEE J-SAC, 22(1):41-53, January 2004.

9. Biggs Norman L. Algebraic Graph Theory. Cambridge Math. Library, 1974, 1993 (2nd edition), 1993.

10. V. Paxson, M. Allman. Computing TCP's Retransmission Timer. Request for Comments: 2988, November 2000.

11. Krishna P. Gummadi, Richard J. Dunn, Stefan Saroiu, Steven D. Gribble, Henry M. Levy, and John Zahorjan. Measurement, modeling, and analysis of a peer-to-peer file-sharing workload. In SOSP '03: Proceedings of the nineteenth ACM symposium on Operating systems principles, pages 314-329, New York, NY, USA, 2003. ACM Press. 